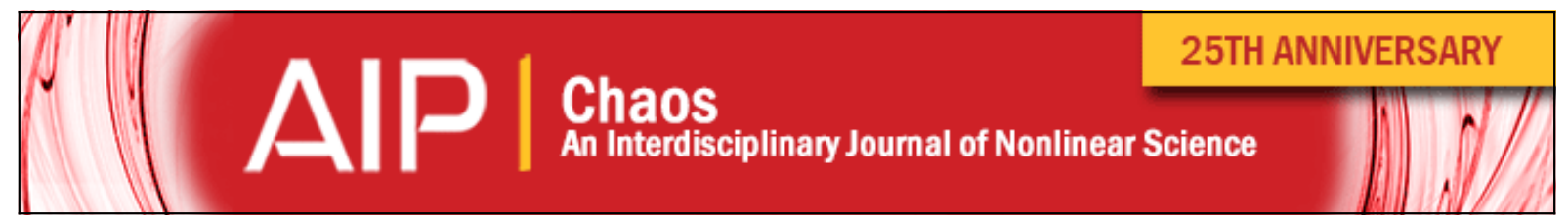

\title{
Chaos in spacecraft attitude motion in Earth's magnetic field
}

V. V. Beletsky, R. V. F. Lopes, and M. L. Pivovarov

Citation: Chaos 9, 493 (1999); doi: 10.1063/1.166422

View online: http://dx.doi.org/10.1063/1.166422

View Table of Contents: http://scitation.aip.org/content/aip/journal/chaos/9/2?ver=pdfcov

Published by the AIP Publishing

\section{Articles you may be interested in}

Spacecraft attitude pulse-width control at initial, service and emergency modes

AIP Conf. Proc. 1493, 933 (2012); 10.1063/1.4765599

Bifurcation and chaos in spin-valve pillars in a periodic applied magnetic field

Chaos 19, 043111 (2009); 10.1063/1.3258365

Design and prototyping of a micropropulsion system for microsatellites attitude control and orbit correction

J. Vac. Sci. Technol. B 20, 2793 (2002); 10.1116/1.1523015

Low-stiction magnetic bearing for satellite application

J. Appl. Phys. 91, 6994 (2002); 10.1063/1.1452673

The $\mu$ TORQUE momentum-exchange tether experiment

AIP Conf. Proc. 608, 299 (2002); 10.1063/1.1449738

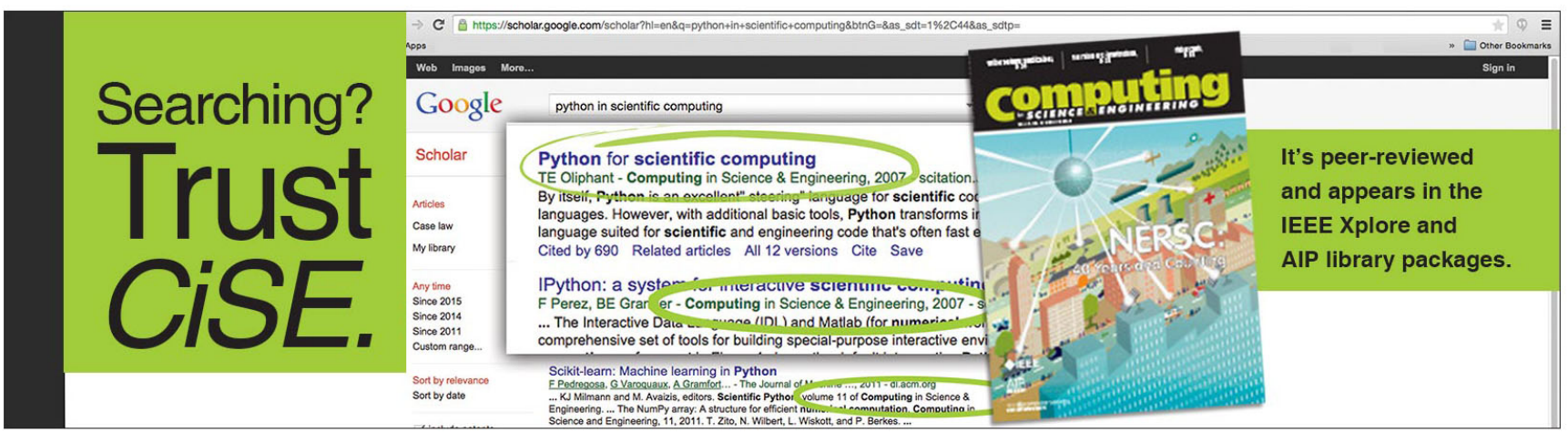




\title{
Chaos in spacecraft attitude motion in Earth's magnetic field
}

\author{
V. V. Beletsky \\ Keldysh Institute of Applied Mathematics, 4 Miusskaya, 125047 Moscow, Russia \\ R. V. F. Lopes \\ National Institute for Space Research-INPE, 12.227-019 S. J. dos Campos SP, Brazil \\ M. L. Pivovarov \\ Space Research Institute, 84/32 Profsoyuznaya, 117810 Moscow, Russia
}

(Received 25 June 1998; accepted for publication 4 January 1999)

\begin{abstract}
The rotational motion of a satellite with a magnetic stabilization system is discussed. The motion is described by a nonautonomous differential equation, with the magnetic moment of the satellite as a parameter. The global phase portrait of the problem is investigated in a wide range of magnetic-parameter values, using a numerical realization of the method of Poincare point maps. New periodic solutions of the problem are found, and an analysis is carried out of the evolution of the phase portrait and the bifurcation of periodic solutions with varying magnetic-parameter values. The values of the magnetic parameter that must be avoided in the design of the satellite magnetic stabilization system are discussed. (C) 1999 American Institute of Physics.

[S1054-1500(99)01402-0]
\end{abstract}

The rotation of a satellite with a magnetic stabilization system is discussed in many publications because of its importance in space research. These publications are largely confined to the analysis of periodic motions. It turns out that the problem is also of interest from the standpoint of the global phase portrait, the resulting bifurcations of periodic solutions, and the investigation of chaotic motion. These questions are also discussed in the present paper.

\section{INTRODUCTION}

The rotational motion of an artificial Earth satellite is examined in the plane of a polar circular Kepler orbit. The satellite is assumed to carry a permanent magnet, used for the stabilization of the spacecraft along the Earth's magnetic lines of force. The magnet axis points along the central principal axis of inertia of the satellite and lies in the plane of the orbit. Only the magnetic moment is taken into account in the analysis of the motion of the satellite center of mass.

The equation of motion for this problem was obtained independently by Fishell ${ }^{1}$ and Beletsky. ${ }^{2}$ It has been the subject of numerous investigations, because despite the above simplifying assumptions, it describes the essential features of the motion and is important in the investigation of the rotational motion of a satellite with a magnetic stabilization system.

Most publications concerned with the above problem deal with regular motions. This is justified by the fact that periodic and quasiperiodic motion are of interest in connection with the design of magnetically stabilized satellites. On the other hand, it is well known that the regular motions of a mechanical system are in general confined to a portion of phase space and are local among chaotic motions. The research reported here is largely confined to the numerical analysis of the global structure of the phase space of the above problem, using the method of Poincaré point maps.

\section{PERIODIC OSCILLATIONS}

The equation of motion for the problem takes the form ${ }^{2}$

$$
\frac{d^{2} \varphi}{d u^{2}}+\alpha \sqrt{1+3 \sin ^{2} u} \sin \varphi=\frac{6 \sin 2 u}{\left(1+3 \sin ^{2} u\right)^{2}},
$$

where $\varphi$ is the angle between the magnetic moment vector $M$ of the satellite and the geomagnetic field $B, u$ is the angle between the plane of the Earth's equator and the radius vector of the satellite, and $\alpha$ is a dimensionless constant that depends on the magnetic moment of the satellite and the moment of inertia of the satellite relative to the principal axis that is perpendicular to the plane of the orbit (see Fig. 1).

Numerous authors have investigated the above equation since it was originally formulated in Refs. 1 and 2. The basic results concerned with the analysis of periodic oscillations were obtained in Refs. 2-8, which were largely confined to the $\pi$-periodic solutions of the form $\varphi(0)=\varphi(\pi / 2)=0$. Some solutions have been found ${ }^{6}$ analytically, but the global analysis of the existence and stability of periodic solutions can only be performed by numerical methods. An asymptotic investigation of the problem is performed in Refs. 9 and 10 for large values of $\alpha$.

Our aim was to extend these results to values of $\alpha$ up to 100 (since the magnetic parameter of real satellites is of the order 70-100) and to investigate the stability of the resulting $\pi$-periodic oscillations in this wider range of parameter values.

The entire analysis was performed numerically, using MATLAB. The initial velocity $\varphi^{\prime}(0)$ was specified for each value of $\alpha$ on the interval $(0,100)$ in steps 0.05 . This velocity is the zero of the function $f=(\varphi(\pi))^{2}+\left(\varphi^{\prime}(\pi)-\varphi^{\prime}(0)\right)^{2}$. Initially, $\varphi(0)=0$. This means that we investigated a some- 


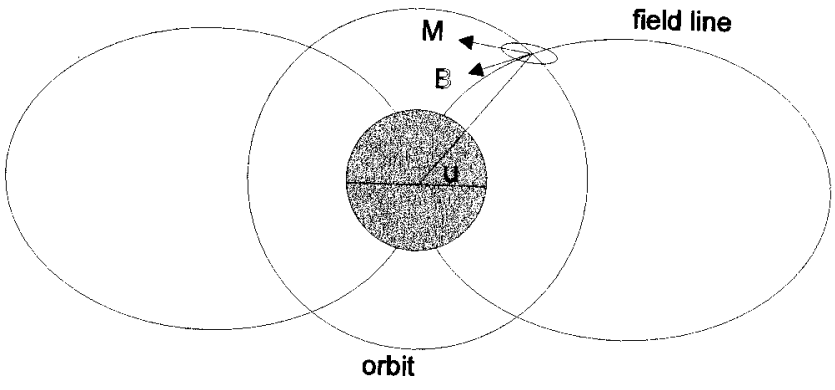

FIG. 1. Description of motion.

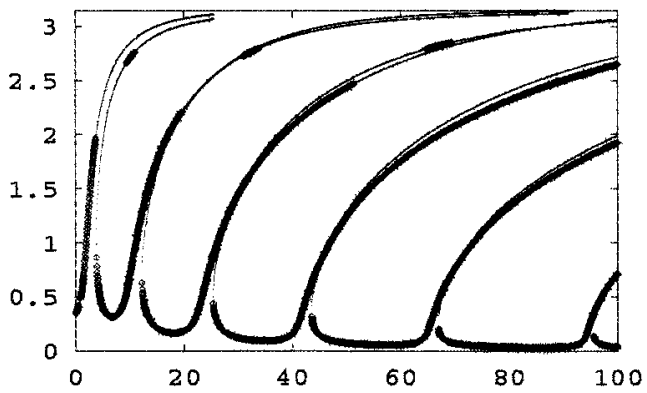

FIG. 2. Amplitude curves.

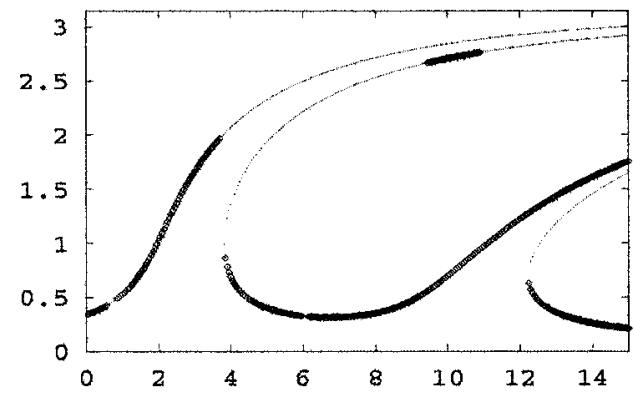

FIG. 3. Fragment of amplitude curves.

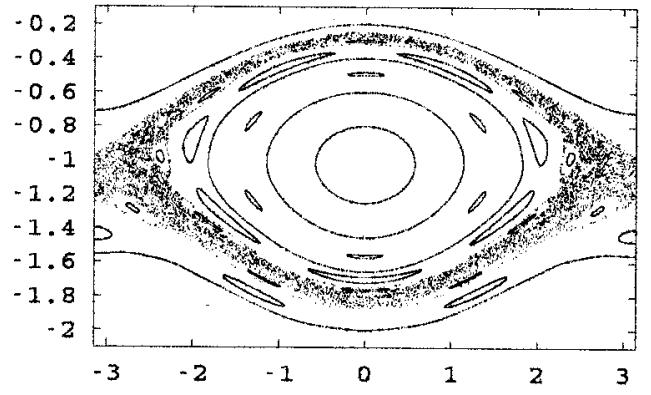

FIG. 4. $\alpha=0.1$. what expanded class of possible $\pi$-periodic oscillations. The oscillation amplitude was computed in this way for each periodic solution. Figures 2 and 3 show the amplitudes (in radians) of all the $\pi$-periodic solutions as functions of the dimensionless parameter $\alpha$. The thicker portions of these curves correspond to stable solutions. For example, for $\alpha$ $=80$ there are nine $\pi$-periodic oscillations of the satellite about a magnetic line of force. Three of them are stable. All branches of the amplitude curves approach asymptotically the straight line that corresponds to the oscillation amplitude equal to $\pi$, at $\alpha \rightarrow \infty$. Portions of these curves that approach this line are not shown in Figs. 2 and 3.

The stability of all solutions was investigated by the method of Poincaré point maps and the results are discussed in Sec. III.

It is clear from Fig. 3 that the amplitude curves display small gaps near $\alpha=0.6$ and 6 . These points correspond to period doubling bifurcation: the $\pi$-periodic oscillations lose their stability and $2 \pi$-periodic stable solutions arise. These bifurcations are examined next.

\section{GLOBAL STRUCTURE OF PHASE SPACE AND STABILITY OF PERIODIC SOLUTIONS}

The investigation reported here is a continuation of the study of regular and chaotic motions in the system (1) that began in Refs. 11-13.

We used a numerical implementation of the method of Poincaré point maps, i.e., Eq. (1) was integrated numerically for different initial values $\left[\varphi(0), \varphi^{\prime}(0)\right]$ and maps were constructed for the points $\left[\varphi(n \pi), \varphi^{\prime}(n \pi)\right), n=1,2 \ldots$ Figures 4-25 show the Poincare point maps of (1) on the $\varphi^{\prime}(\mathrm{rad} / \mathrm{s}), \varphi(\mathrm{rad})$ plane for different values of the parameter $\alpha$.

In accordance with the method of Poincare point maps, fixed points of the map correspond to periodic solutions, regular curves correspond to quasiperiodic solutions, and irregularly distributed points correspond to chaos.

As in the case of the pendulum, all the phase portraits are bounded by regular curves that correspond to the rotation of the satellite about the magnetic line of force. The angular velocities of these rotations range from $0.2 \mathrm{rad} / \mathrm{s}$ for $\alpha \approx 0.1$ (Fig. 4) to $25 \mathrm{rad} / \mathrm{s}$ for $\alpha \approx 100$ (Fig. 24). Periodic oscillations are confined to this range, which means that the Poincaré map gives the boundaries of the range of initial-velocity values in which the periodic oscillations may be found.

The relative dimensions of the chaotic region attain a minimum for very low and very high values of $\alpha$ (Figs. 4 and 24 , respectively). Chaotic motions predominate for $\alpha \approx 0.8$. On the other hand, very low and maximum values of $\alpha$ correspond to the maximum relative size of the region of regular motion. The center of this region has zero $x$-coordinate. The centers of the regions of regular motion with the $x$-coordinate equal to $\pi$ correspond to stable $\pi$-periodic rotation about the magnetic line of force. Bearing in mind possible applications to magnetic stabilization of artificial Earth satellites, we were above all interested in the analysis of $\pi$-periodic oscillations. Islands in the chaotic sea correspond to long-period solutions. For example, Fig. 5 shows $3 \pi$ - 


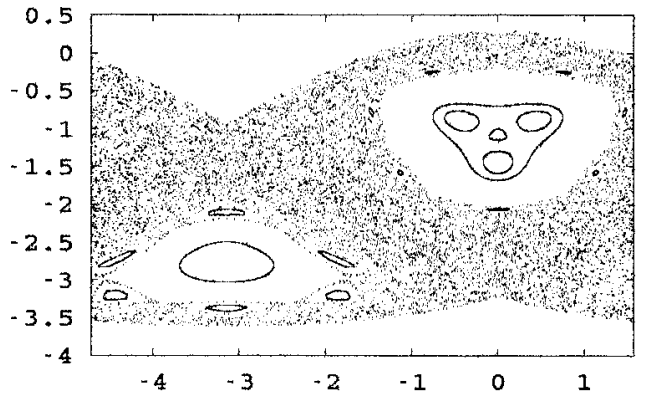

FIG. 5. $\alpha=0.3$.

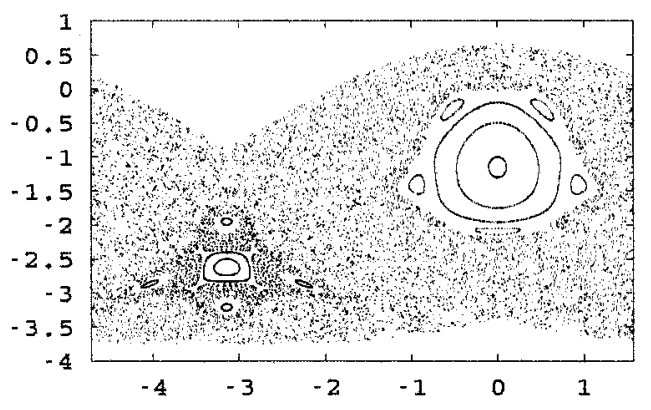

FIG. 6. $\alpha=0.5$.

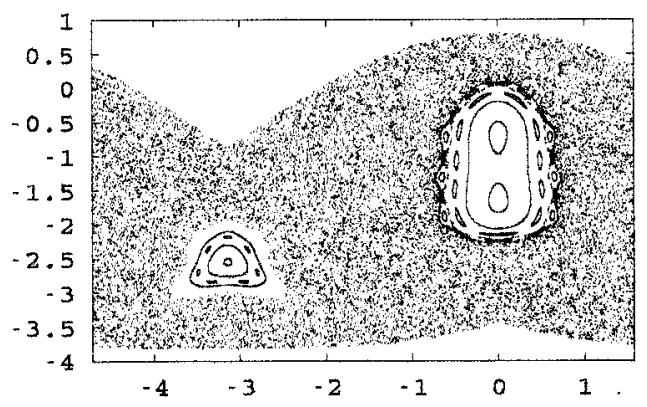

FIG. 7. $\alpha=0.6$.

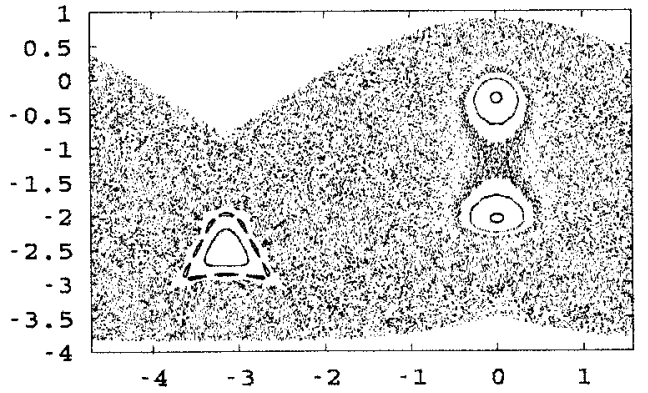

FIG. 8. $\alpha=0.66$.

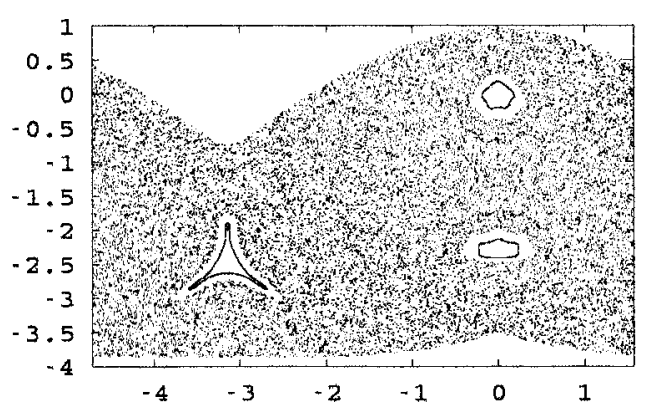

FIG. 9. $\alpha=0.7$.

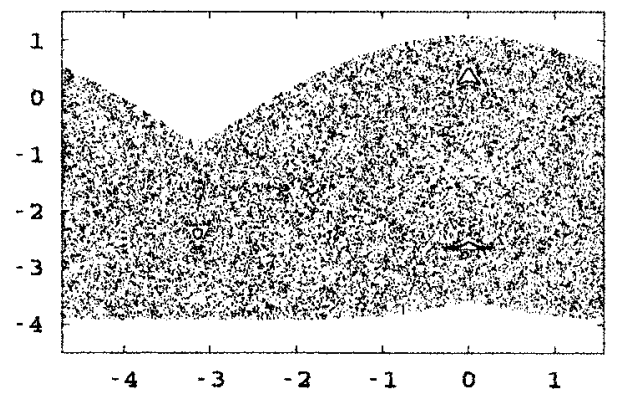

FIG. 10. $\alpha=0.8$.

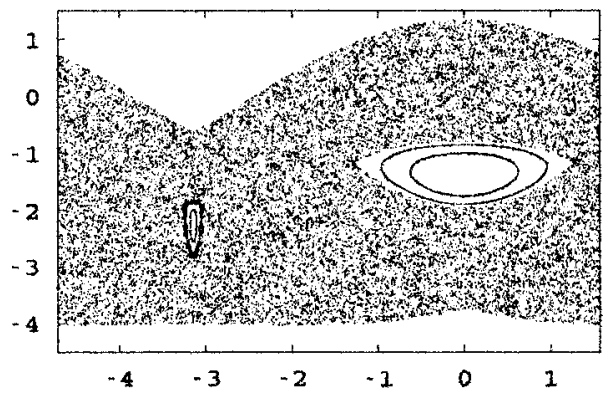

FIG. 11. $\alpha=1$.
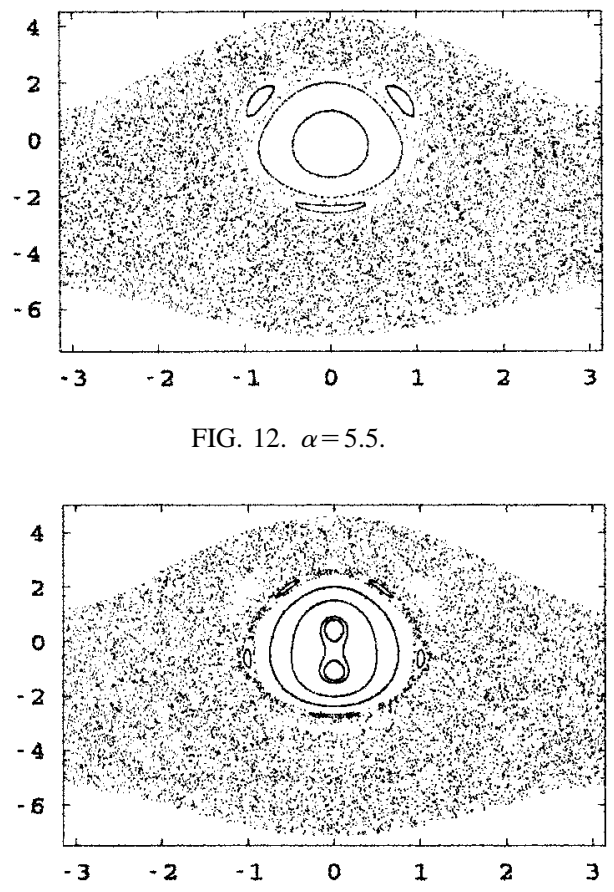

FIG. 13. $\alpha=6$.

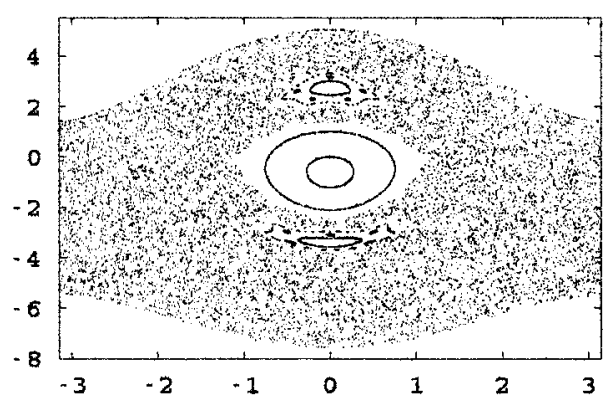

FIG. 14. $\alpha=7$. 


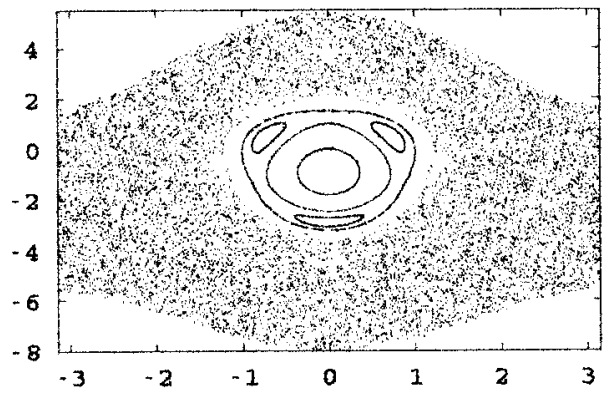

FIG. 15. $\alpha=8$.

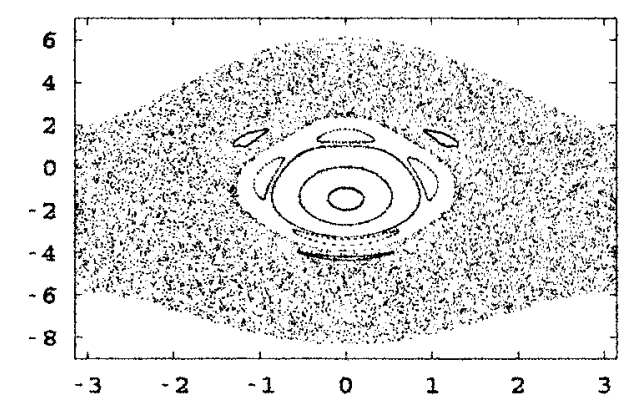

FIG. 16. $\alpha=9$.

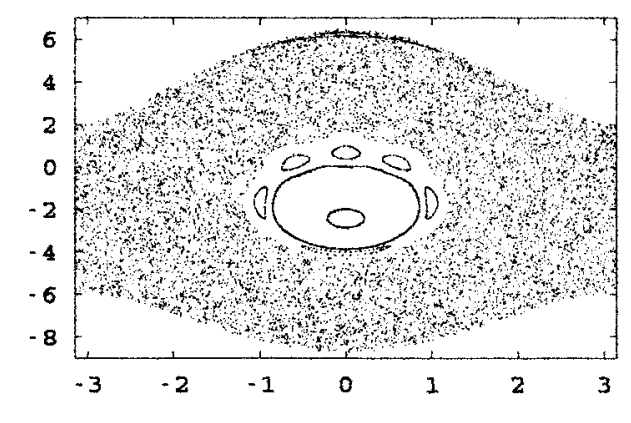

FIG. 17. $\alpha=10$.

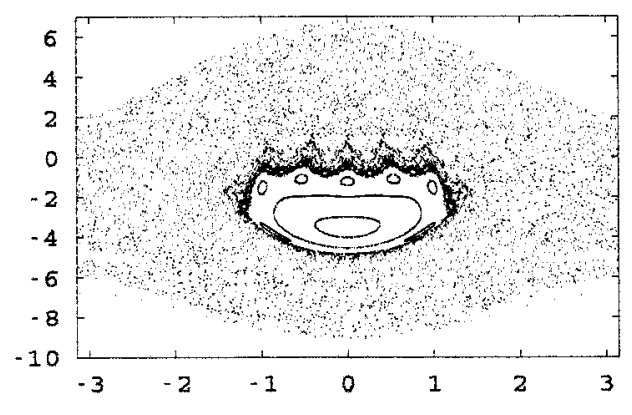

FIG. 18. $\alpha=11$.

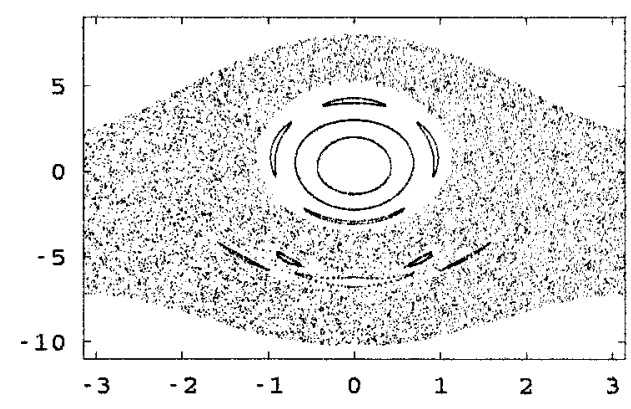

FIG. 19. $\alpha=15$.

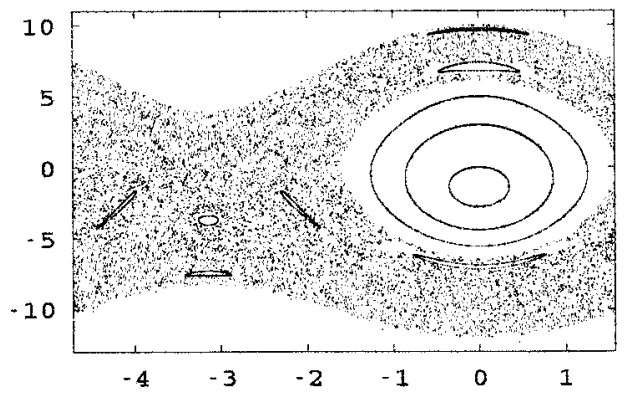

FIG. 20. $\alpha=22.5$.
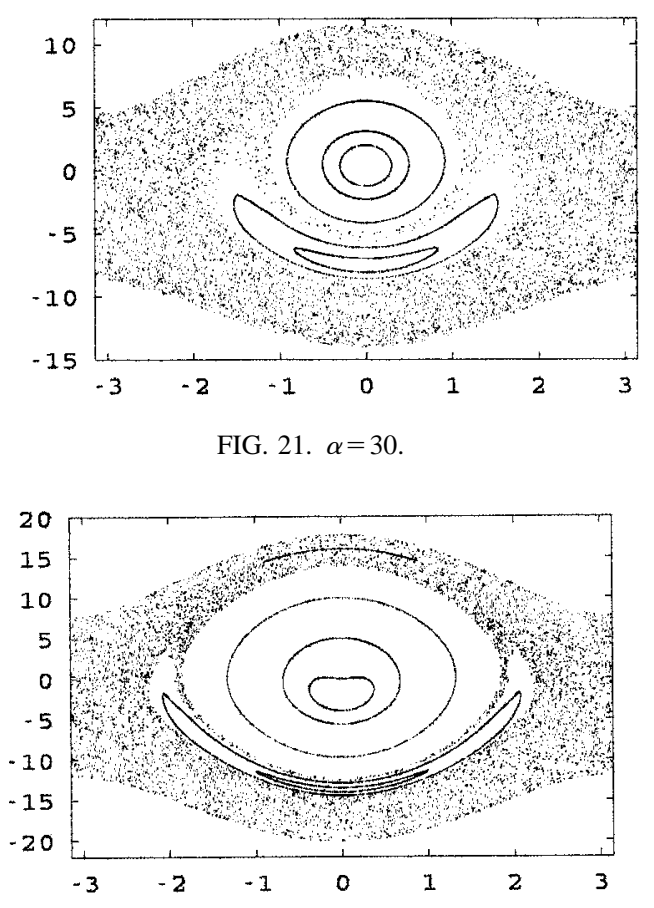

FIG. 22. $\alpha=66$.

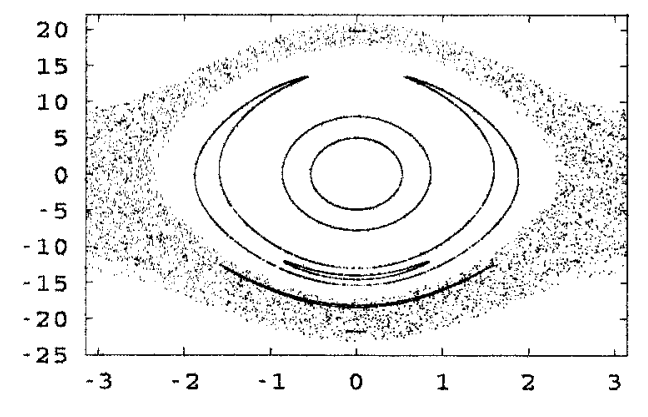

FIG. 23. $\alpha=90$.

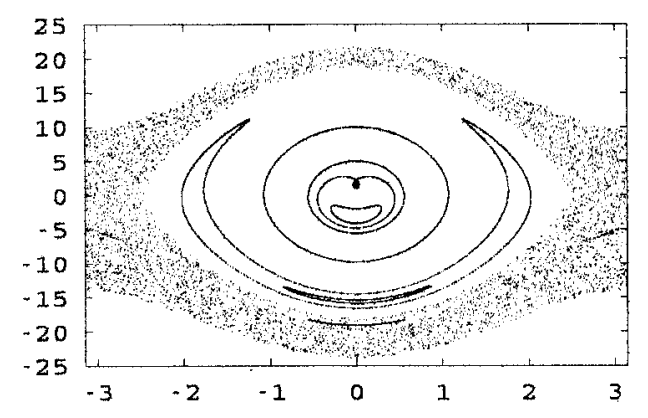

FIG. 24. $\alpha=95.55$. 


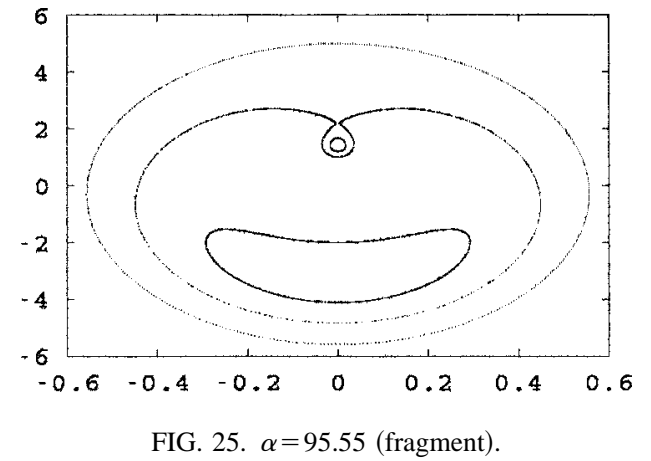

periodic solutions around the $\pi$-periodic solution, while Fig. 7 shows the $11 \pi$-periodic solution around the $2 \pi$-periodic solution.

Figures $6-8$ show period-doubling bifurcations ${ }^{14}$ near $\alpha=0.6$. In accordance with the period-doubling scenario for Hamiltonian systems [the system described by (1) takes the Hamiltonian form when expressed in terms of certain variables], the $\pi$-periodic trajectory loses its stability and a stable $2 \pi$-periodic trajectory splits from it. Figures 12 and 13 show this type of bifurcation near $\alpha=6$.

It is clear from Figs. 2 and 3 that there are values of $\pi$ that are the points of creation of a new branch of the amplitude curve, i.e., points of creation of a new pair of $\pi$-periodic solutions: a stable solution and an unstable one. The amplitude of stable oscillations of the satellite can rise near these values of $\alpha$, and such values should be avoided when the magnetic stabilization system of a satellite is designed. These resonance values of $\alpha$ were found analytically in Ref. 3 . They are: $2.5,10,22.5,40,62.5,90$, and so on.

Figure 25 illustrates the creation of a pair of $\pi$-periodic oscillations near $\alpha=95$.

The center of the smallest closed curve in Fig. 25 (its $y$-coordinate is $1.41 \ldots)$ corresponds to a new stable $\pi$-periodic solution. The point with the $y$-coordinate of 2.19 ... corresponds to a new unstable $\pi$-periodic solution.

To analyze the stability of the $\pi$-periodic solutions that we have found, we used a nonrigorous approach based on the method of Poincaré point maps. It is well known that stable and unstable solutions correspond to fixed points on the map. However, a small uncertainty in the initial conditions for stable motion leads to a small regular curve around a fixed point. On the other hand, the uncertainty in the initial conditions for unstable motion leads either to a regular curve around another stable periodic solution or to chaos.

In other words, if in the course of a substantial number of mappings the phase point remains in a "small" neighborhood of the initial point, it is reasonable to assume that the initial point corresponds to a stable periodic solution; otherwise the solution is unstable.

This nonrigorous approach was used to examine the stability of all our $\pi$-periodic oscillations $\alpha \in(0,100)$. The thick portions of the curves in Figs. 2 and 3 correspond to stable oscillations.

Our results agree with rigorous determinations in the limited range of magnetic-parameter values in which stabil- ity was investigated rigorously, and may be useful when rigorous methods are employed.

We also note that our numerical investigation was concerned with a special class of $\pi$-periodic oscillations of the satellite, namely $\varphi(0)=0$. These and only these solutions were found on the Poincare point maps. However, there is no certainty as to the existence of other $\pi$-periodic oscillations.

\section{COMPARISON OF THE CHAOTIC REGION WITH ITS THEORETICAL BOUNDARY IN THE ADIABATIC APPROXIMATION}

Equation (1) describes the motion of a satellite about its center of mass in the coordinate frame attached to the magnetic field vector. We shall now use another form of this equation. In particular, we shall consider the motion of a satellite in a uniformly rotating coordinate frame $O X Y$ whose origin $O$ coincides with the position of the satellite on an orbit corresponding to the angle $u$; the $X$-axis is at an angle $2 u$ to the plane of the equator and the $Y$-axis makes an angle $2 u$ with the Earth's axis of rotation.

It is readily shown that, in this coordinate frame, the geomagnetic field vector oscillates about the $Y$-axis, where the angle of deflection of this axis is

$$
\nu=\arcsin \left(-\sin (u) \cos (u) / \sqrt{1+3 \sin ^{2}(u)}\right) .
$$

Let $\psi$ be the angle between the axis of the satellite along which the magnet is mounted and the $Y$-axis of the above reference frame. The equation of rotation of the satellite then takes the form

$$
\frac{d^{2} \psi}{d u^{2}}+\alpha \sqrt{1+3 \sin ^{2}(u)} \sin (\psi+\nu(u))=0 .
$$

When $\alpha \gg 1$, and if we put $u=z \sqrt{\alpha}$, Eq. (2) reduces to the equation of a pendulum with slowly varying frequency and a phase shift. This means that the results reported in Refs. 15 and 16 are valid for Eq. (2). The theoretical boundaries of the chaotic region can be constructed for Eq. (2) in the following manner. The boundary between the chaotic region and the region of regular rotation in the forward direction is the rotational trajectory of a pendulum of fre-

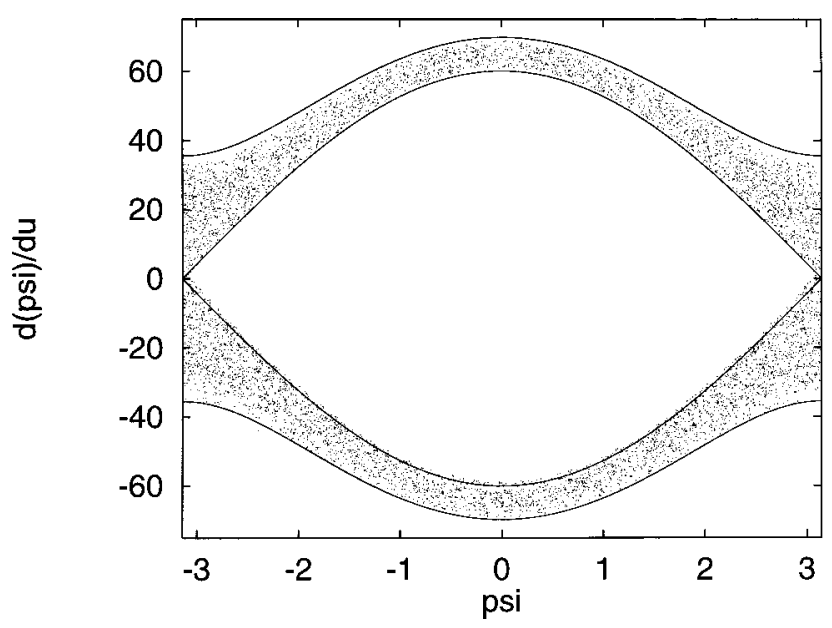

FIG. 26. Comparison of chaotic region with its theoretical boundary. 
quency $\sqrt{\alpha}$, the area under the trajectory being equal to the maximum area under the separatrix of the pendulum (2), i.e., under the separatrix of a simple pendulum of frequency $\sqrt{2 \alpha}$. The rotational trajectory that is symmetric relative to the abscissa axis is the boundary between the chaotic region and the region of regular rotation in the reverse direction. The inner boundary of the chaotic region is the separatrix of the simple pendulum of frequency $\sqrt{\alpha}$.

Figure 26 shows the chaotic region of Eq. (2) for $\alpha$ $=900$ (i.e., when the magnitude of the small parameter is $1 / 30$ ), obtained by the method of Poincare point maps for the trajectory with the initial point $(-\pi, 20)$ and $u=0$. The regular curves are the boundaries discussed above. It is clear that the chaotic region extends somewhat beyond the theoretical boundary represented by the lower branch of the separatrix.

Small islands of stability ${ }^{17}$ may exist within the chaotic region.

\section{ACKNOWLEDGMENTS}

The authors are indebted to A. I. Neishtadt for useful discussions. This research was supported by FAPESP (process No. 1996/3247-0/INPE Brazil), and also partially by Grant RFBR-98-01-00940 and the Astronomy Program (section 1.7.4.1) of the Russian Federation.
${ }^{1}$ R. E. Fishell, "Magnetic and gravity attitude stabilization of Earth satellites," in Space Research II Proc. of the Second Intern. Space Science Symposium, Florence, April 10-14, 1961 (Amsterdam, 1961), pp. 373410.

${ }^{2}$ V. V. Beletsky, The Motion of an Artificial Satellite about its Center of Mass (in Russian) (Nauka, Moscow, 1965).

${ }^{3}$ V. V. Beletsky, Studies on the Motion of Space Bodies (in Russian) (Nauka, Moscow, 1977).

${ }^{4}$ V. V. Beletsky and A. N. Shlyachtin, Preprint No. 46, Keldysh Institute of Applied Mathematics, USSR Academy of Sciences, 1980.

${ }^{5}$ V. V. Beletsky and A. A. Khentov, Magnetized Spacecraft Rotation (in Russian) (Nauka, Moscow, 1985).

${ }^{6}$ A. A. Khentov, Kosm. Issled. 5, 540 (1967).

${ }^{7}$ M. Yu. Ovchinnikov, "Magnetized spacecraft periodic oscillations on the plane of a circular polar orbit," in Aerophysics and Applied Mathematics (MFTI, Moscow, 1980).

${ }^{8}$ V. A. Sarychev and M. Yu. Ovchinnikov, Magnetic Orientation Systems for Artificial Earth's Satellites (in Russian) (VINITI, Moscow, 1985).

${ }^{9}$ M. L. Pivovarov, Kosm. Issled. 24, 816 (1986).

${ }^{10}$ M. L. Pivovarov, "Magnetically stabilized spacecraft attitude motion and differential equations with slowly changing coefficients," in Proceedings of Third International Symposium on Spacecraft Flight Dynamics, Darmstadt, Germany, 1991.

${ }^{11}$ Z. S. Batalova and N. A. Mel'nichenko, Kosm. Issled. 21, 522 (1983).

${ }^{12}$ V. V. Beletsky, Regulaere und Chaotische Bewegung Starrer Koerper (in German) (Teubner-Verlag, Stuttgart, 1995).

${ }^{13}$ V. V. Beletsky, M. L. Pivovarov, and E. L. Starostin, Chaos 6, 155 (1996).

14 “'Dynamical systems," in Encyclopaedia of Mathematical Sciences, edited by V. I. Arnold (Springer-Verlag, Berlin, 1994), Vol. 5.

${ }^{15}$ A. I. Neishtadt, Sov. J. Plasma Phys. 12, 568 (1986).

${ }^{16}$ J. L. Tennyson, J. R. Cary, and D. F. Escande, Phys. Rev. A 34, 4526 (1986).

${ }^{17}$ A. I. Neishtadt, V. V. Sidorenko, and D. V. Treshev, Chaos 7, 2 (1997). 